\title{
An EOQ Model with Time Dependent Weibull Deterioration, Quadratic Demand and Partial Backlogging
}

\author{
Umakanta Mishra ${ }^{1}$
}

Published online: 26 July 2015

(C) Springer India Pvt. Ltd. 2015

\begin{abstract}
This paper presents an order level inventory system with time dependent Weibull deterioration and quadratic demand rate where holding costs as a linear function of time. The proposed model considered here to allows for shortages, and the demand is partially backlogged. The model is solved analytically by minimizing the total inventory cost. The implementation of the proposed model is illustrated using some numerical examples. Sensitivity analysis is performed to show the effect of changes in the parameters on the optimum solution.
\end{abstract}

Keywords EOQ · Webull deterioration · Shortage $\cdot$ Demand $\cdot$ Holding cost

\section{Introduction}

In the traditional inventory model, the demand rate is assumed to be constant. This happens very rarely in realistic situations same inventory model have been developed for dealing with the time varying demand. The quadratic demand technique is applied to control the problem in order to determine the optimal production policy. Quadratic demand seems to be a better representation of time varying market demands. Some researchers suggest that rapidly increasing demand can be represented by an exponential function of time. The assumption of an exponential rate of change in demand is high and the fluctuation or variation of any commodity in the real market cannot be so high. Thus, this accelerated growth in demand rate in the situations like demands of computer chips of computerized machines, spare parts of new aeroplanes etc. is changing the demand more rapidly. Therefore, this situation can be best represented by a quadratic function of time. Some researchers consider the demand rate functions in the form of linear demand as $D(t)=a+b t, a \geq 0, b \neq 0$ or exponential type demand rate like $D(t)=\alpha e^{\beta t}, \alpha>0, \beta \neq 0$. The linear type demand show steady

Umakanta Mishra

umakanta.math@gmail.com

1 Faculty of Science and Technology, ICFAI University, Tripura, Kamalghat,

Sadar, West Tripura 799210, India 
increase $(b>0)$ or decrease $(b<0)$ in the demand rate, which is rarely seen in real market. Also the exponential rate is being very high, i.e. it increases $(\beta>0)$ or decreases $(\beta<0)$ exponentially with the demand rate. Therefore the real market demand of any product may rise or fall exponentially. The demand rate functions of the form $D(t)=a+b t+c t^{2}, a \geq$ $0, b \neq 0, c \neq 0, \frac{d D(t)}{d t}=b+2 c t, \frac{d^{2} D(t)}{d t^{2}}=2 c$.

Then we have the following cases depending on

(a) For $b>0$ and $c>0$, the rate of increase of demand rate $D(t)$ is itself an increasing function of time which is termed as accelerated growth in demand.

(b) For $b>0$ and $c<0$, there is retarded growth in demand for all time.

(c) For $b<0$ and $c<0$, the demand rate $D(t)$ decreases at a decreasing rate which we may call it as accelerated decline demand. This case usually happens to the spare parts of an obsolete aircraft model or microcomputer chip of high technology products substituted by another.

(d) For $b<0$ and $c>0$, the demand rate falls at an increasing rate for $t>\left(-\frac{b}{2 c}\right)$.

Thus we may have different types of realistic demand patterns from the functional form $D(t)=a+b t+c t^{2}$ depending on the signs of $b$ and $c$. Therefore the quadratic timedependence of demand is more realistic than its linear or exponential time-dependent demand. Time-dependent quadratic demand Khanra and Chaudhuri [9], explains the accelerated growth/decline in the demand patterns. Depending on the signs of $\mathrm{b}$ and $\mathrm{c}$ we may explain different types of realistic demand patterns. Demands for spare parts of new aeroplanes, computer chips of advanced computer machines, etc. increased widely while the demands for spare parts of the obsolete aircrafts, computer chips etc. decreased very rapidly with time. These concepts addressed well in the inventory models with quadratic demand rate, like ([1,6,10,18,20,24,25] etc.) have been devoted to incorporating a quadratic demand rate into their models with or without shortages under a variety of circumstances.

In formulating inventory models, the deterioration of items should not be neglected for all items. For example, items like foodstuff, pharmaceuticals, chemicals, etc., deteriorate significantly. Many researchers like ([2,5,17,22,23,26] etc.), assumed, for simplicity, that items deteriorate at a constant rate. The assumption of constant deterioration rate was relaxed by Covert and Philip [3]. They used a two parameter Weibull distribution to represent the distribution of time to deteriorate. This model was further generalized by Philip [15], by taking three-parameter Weibull distribution [14] adopted a two parameter Weibull distribution deterioration to develop an inventory model with finite rate of replenishment. These investigations were followed by $([4,8,19]$ etc.), where the deterioration rate was considered to be time-proportional.

In the above mentioned references, most researchers assumed that shortages are completely backlogging. In practice, some customers would like to wait for backlogging during the shortage period, but the other would not. Consequently, the opportunity cost due to lost sales should be considered in the model. Taking these concepts many research works $([7,11-$ $13,16,21]$ etc.).

The work of the researchers who used quadratic demand function and various forms of deterioration with shortages (allowed/not allowed), for developing the economic order quantity (EOQ) models are summarized below:

The above Table 1 shows that all the researchers developed EOQ models by taking quadratic demand, deterioration (Constant/Linear/Weibull distribution) and shortages (allowed/not allowed), and holding costs as constant. But holding cost may not be constant over time, as there is a change in time value of money and change in the price index. The 


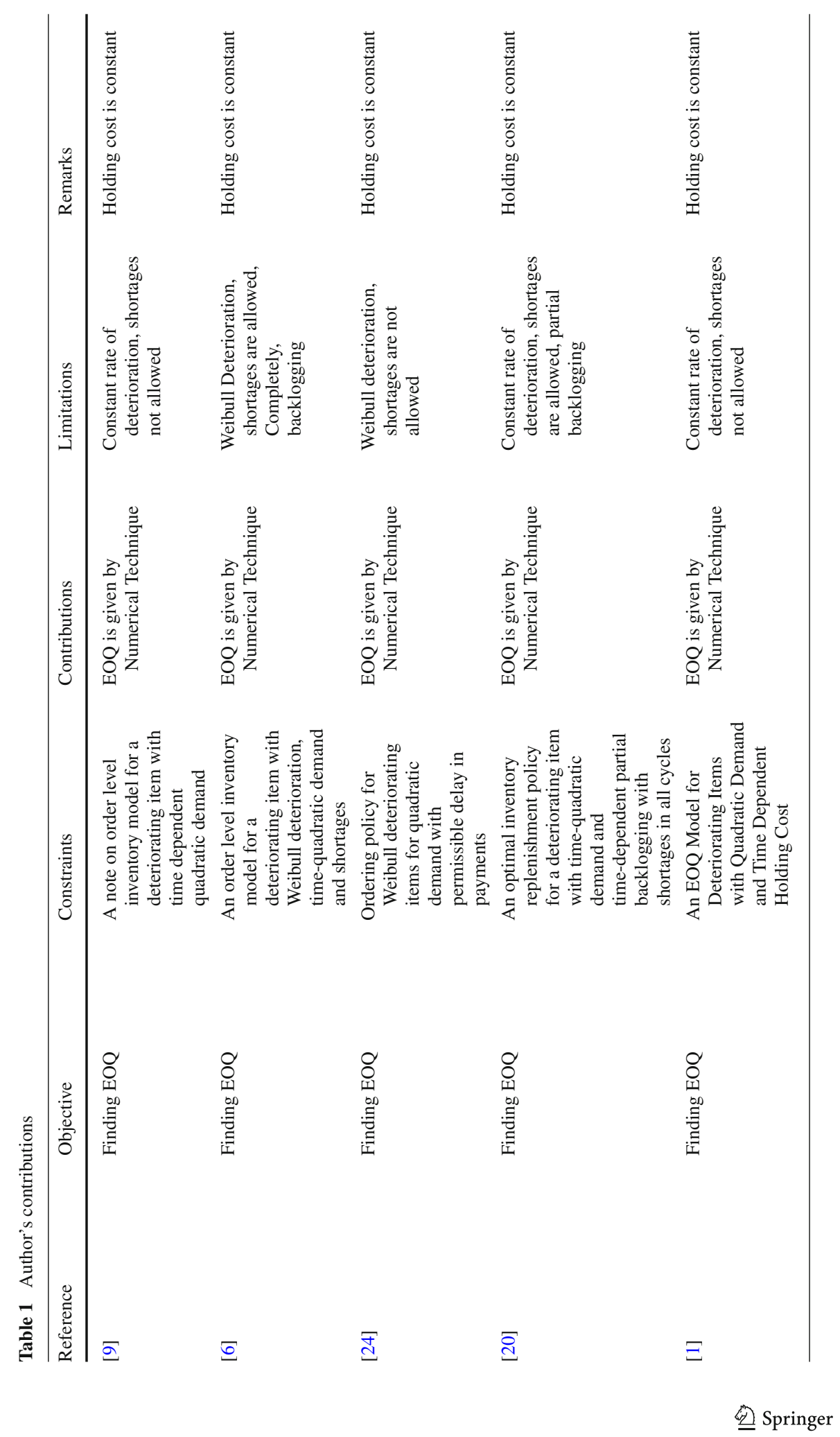




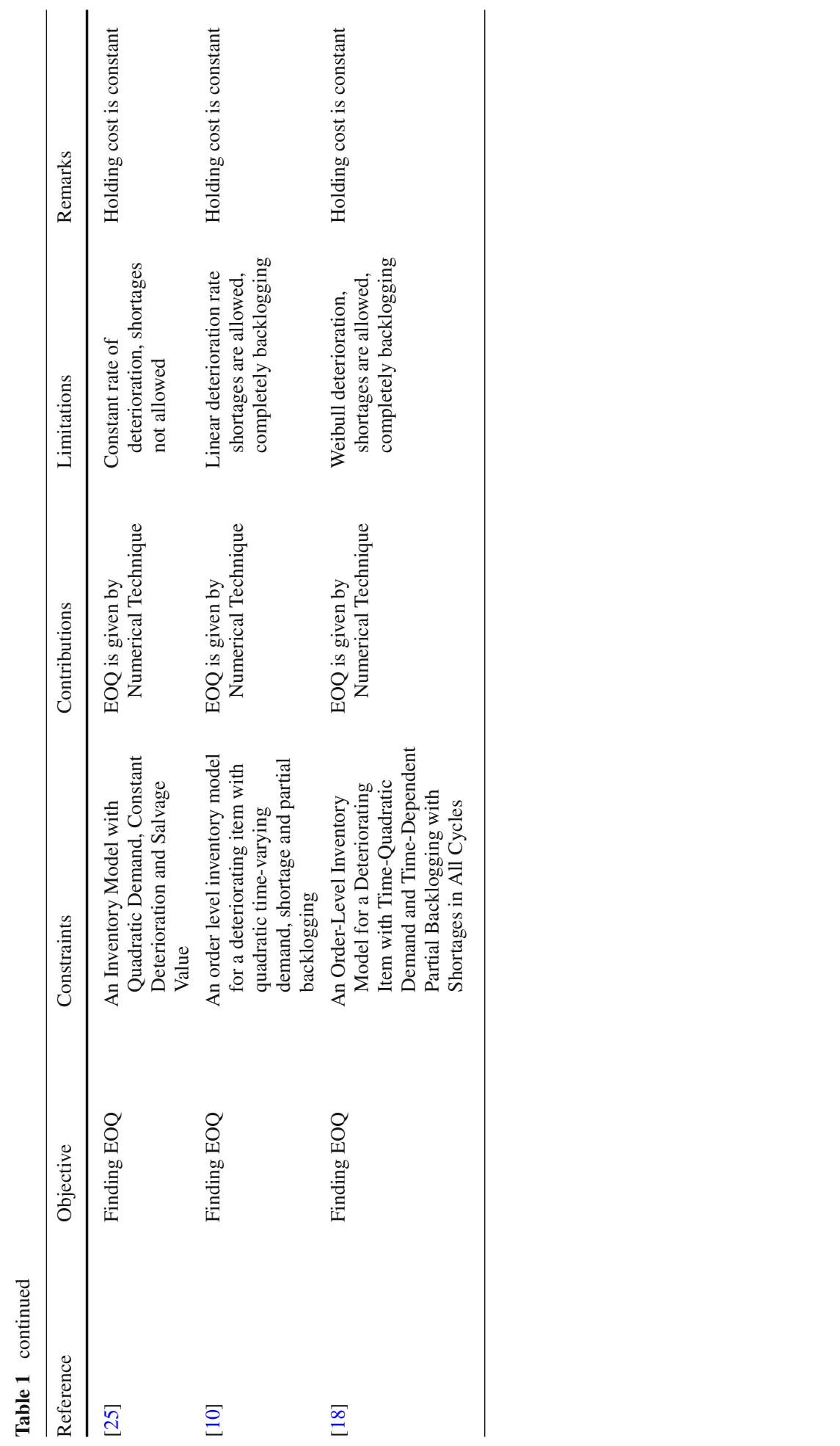


motivation behind developing an inventory model in the present article is to prepare a more general inventory model, which includes; (a) Holding cost as a linearly increasing function of time, (b) Deterioration rate as a two parameter Weibull distribution. The main reason for choosing the Weibull distribution deterioration lies in its convenient generalized properties. The novelty we will be taking into consideration in this research is that the time of deterioration is a random variable following the two parameter Weibull distribution. Two-parameter Weibull distribution deterioration is a generalized form of exponentially decaying functions. This distribution can be used to model either increasing or decreasing rate of deterioration, according to the choice of the parameters. Many inventory models have been developed using backlogging rate to be an exponential function of waiting time of the customers, but in real practice, backlogging rate never varies as high as exponential. Therefore, in the present paper considering the backlogging rate to be $\frac{1}{1+\delta x}, \delta>0$, where $x$ is the waiting time of the customers for receiving their goods which seems to be better. This model can be applied to optimize the total inventory cost for the business enterprises where both the holding cost and deterioration rate are time dependent.

In this paper, a new economic order quantity (EOQ) models for deteriorating items which are time dependent and the demand rate is a quadratic. Shortages are allowed and partially backlogged here. The assumptions and notations of the model are introduced in "Notations and Assumption" section. In "Mathematical Formulation and Solution Procedure" section, a mathematical model is established and solution procedure is discussed for minimizing the total cost. Numerical examples are provided to illustrate the proposed model in "Numerical Example" section. The model proved that the total cost function is convex in "Graphical Proof for the Convexity of the Total Cost Function" section. Total cost changes by changing for different values of parameters for the Weibull distribution provided in "Case Study" section. Sensitivity analysis of the optimal solution with respect to major parameters of the system is carried out, and their results are discussed in "Sensitivity Analysis" section. The article ends with some concluding remarks and scope of future research.

\section{Notations and Assumption}

This inventory model is developed on the basis of the following notations and assumption:

\section{Notations}

$\begin{array}{ll}\text { i. } & T=t_{1}+t_{2}: \text { Length of the cycle. } \\ \text { ii. } & t_{1}: \text { the time at which the inventory level reaches zero. } \\ \text { iii. } & t_{2}: \text { the length of period during which shortages are allowed. } \\ \text { iv. } & I(t): \text { the level of positive inventory at time } t . \\ \text { v. } & A: \text { Ordering cost per order. } \\ \text { vi. } & Q: \text { Order quantity of one cycle. } \\ \text { vii. } & c_{1}: \text { Unit cost of an item. } \\ \text { viii. } & c_{2}: \text { Shortage cost per unit per unit time. } \\ \text { ix. } & s: \text { Lost sale cost per unit. } \\ \text { x. } & Q_{0}=I M: \text { Maximum inventory level during }\left(0, t_{1}\right) . \\ \text { xi. } & I B: \text { Maximum inventory level during the shortage period. } \\ \text { xii. } & T A C=\text { Total average cost. }\end{array}$




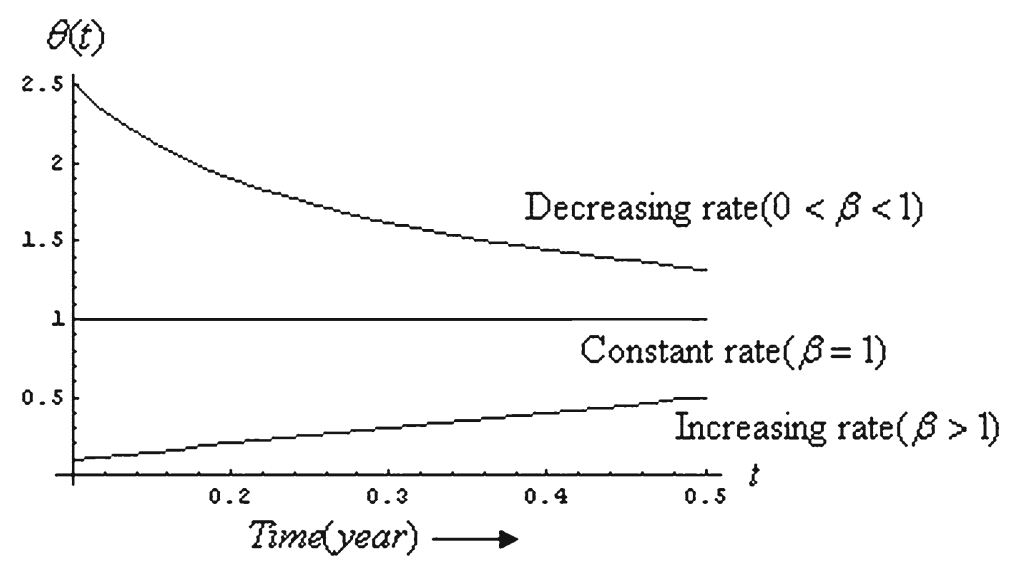

Fig. 1 Rate of deterioration $\theta(t)$ versus time $t$ for a two-parameter Weibull distribution

\section{Assumptions}

i. $\quad$ Lead time is zero.

ii. $\quad D(t)=a+b t+c t^{2}, a \geq 0, b \neq 0, c \neq 0$ : Demand rate is time dependent, where $a$ is initial rate of demand, $b$ is the rate with which the demand rate increases. The rate of change in the demand rate itself increases at a rate $c$.

iii. $\quad h(t)=h+\gamma t$ : Holding cost $h(t)$ per unit time is time dependent, where $h>0$ and $\gamma>0$

iv. $\quad \theta(t)=\alpha \beta t^{\beta-1}$ : Deterioration rate which follows a two parameter Weibull distribution, where $0 \leq \alpha<<1$ is the scale parameter, $\beta>0$ is the shape parameter and $0 \leq \theta(t)<<1$. If $0<\beta<1$ then $\theta(t)$ decreases with time; e.g. a light bulb where the initial deterioration rate may be higher due to irregular voltages and handling. If $\beta=1$, then it is constant, e.g. electronic products, and when $\beta>1$, then it increases with time, e.g. volatile liquids and drugs. Rate of deterioration $\theta(t)$ versus time $t$ for this two parameter Weibull distribution is as shown in Fig. 1. It is seen in Fig. 1 that the two-parameter Weibull distribution is appropriate for an item with a decreasing rate of deterioration only if the initial rate of deterioration is extremely high. Similarly, this distribution can also be used for an item with an increasing rate of deterioration only if the initial rate is approximately zero. This deterioration may also lead to a constant rate of deterioration. However, the case of the increasing rate of deterioration with time is usually seen to hold in all the products and we have considered this case in "Case Study" section.

v. Shortages are allowed. Unsatisfied demand is backlogged, and the fraction of shortages backordered is $\frac{1}{1+\delta x}$, where $x$ is the waiting time up to the next replenishment and $\delta$ is a positive constant. Therefore, if customers do not need to wait, then no sales are lost, and all sales are lost if customers are faced with an infinite wait.

\section{Mathematical Formulation and Solution Procedure}

Given the assumptions mentioned before, the inventory level follows the pattern depicted in Fig. 2. To establish the total relevant cost function, we consider the following time intervals 


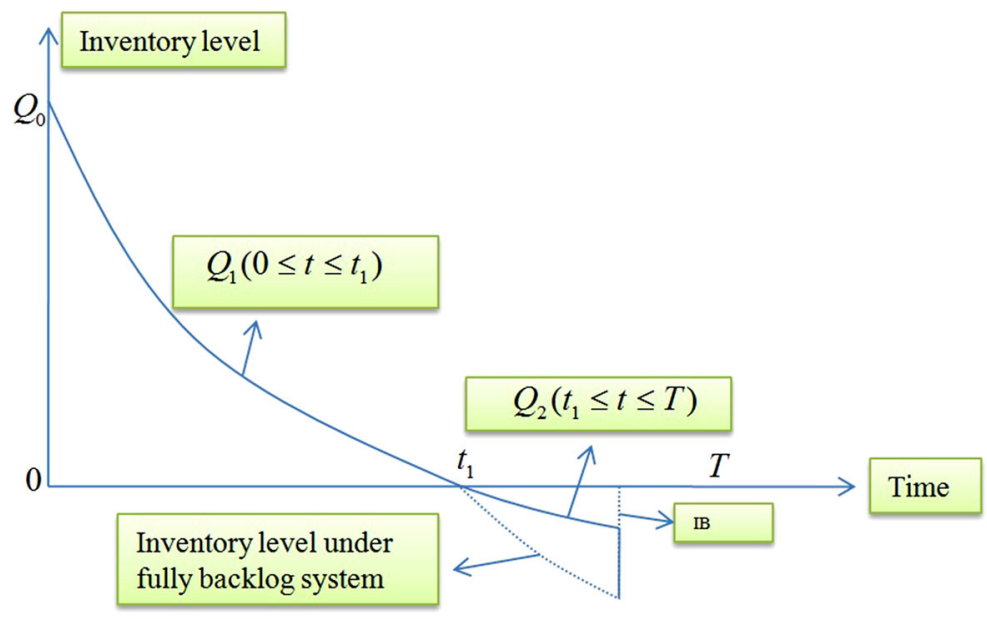

Fig. 2 Graphical representation of the state of inventory system

separately, $\left[0, t_{1}\right)$ and $\left[t_{1}, t_{1}+t_{2}\right)$. During the interval $\left[0, t_{1}\right)$, the inventory is depleted due to the combined effects of demand and deterioration.

Hence, the inventory level is governed by the following differential equation:

$$
\frac{d Q_{1}(t)}{d t}+\theta(t) Q_{1}(t)=-D(t), \quad 0 \leq t \leq t_{1}
$$

with the initial and boundary condition $Q_{1}(0)=Q_{0}$ and $Q_{1}\left(t_{1}\right)=0$.

At time $t_{1}$, the inventory level reaches zero and shortage occurs. During the stock out period, some customers may be willing to wait for a shipping delay while others will leave for another seller because of urgent need. For a customer who desires to purchase the goods at the time $t_{2} \in\left[t_{1}, t_{1}+t_{2}\right),\left(t_{1}+t_{2}-t\right)$ represents the waiting time up to the next replenishment. Hence, over the time interval $\left[t_{1}, t_{1}+t_{2}\right)$, the inventory level depends only on demand, some of which is lost while a fraction $\frac{1}{1+\delta\left(t_{1}+t_{2}-t\right)}$ of the demand is backlogged, where $t \in\left[t_{1}, t_{1}+t_{2}\right)$. The inventory level is governed by the following differential equation:

$$
\frac{d Q_{2}(t)}{d t}=-\frac{D(t)}{1+\delta\left(t_{1}+t_{2}-t\right)}, \quad t_{1} \leq t \leq t_{1}+t_{2}
$$

with the boundary condition $Q_{2}\left(t_{1}\right)=0$.

The solutions of Eq. 1 can be written as follows:

$$
Q_{1}(t)=-e^{-\alpha t^{\beta}} \int_{t}^{t_{1}} e^{\alpha t^{\beta}}\left(a+b t+c t^{2}\right) d t+c e^{-\alpha t^{\beta}}, \quad 0 \leq t \leq t_{1} .
$$

Using boundary condition and expanding by Taylor's series and neglecting small quantities above the rest order, because $\alpha \ll 1$ we get, 


$$
\begin{aligned}
c & =Q_{0} \\
& =\left(a t_{1}+\frac{b t_{1}^{2}}{2}+\frac{c t_{1}^{3}}{3}+\frac{a \alpha t_{1}^{\beta+1}}{\beta+1}+\frac{b \alpha t_{1}^{\beta+2}}{\beta+2}+\frac{c \alpha t_{1}^{\beta+3}}{\beta+3}+2 a \alpha t_{1}^{\beta+1}+b \alpha t_{1}^{\beta+2}+\frac{2 c \alpha t_{1}^{\beta+3}}{3}\right) .
\end{aligned}
$$

Similarly the solution of Eq. (2) can be written as:

$$
\begin{aligned}
Q_{2}(t)= & {\left[\frac{a}{\delta}+\frac{b\left[1+\delta\left(t_{1}+t_{2}\right)\right]}{\delta^{2}}+\frac{c\left[1+\delta\left(t_{1}+t_{2}\right)\right]^{2}}{\delta^{3}}\right] \log \left[\frac{1+\delta\left(t_{1}+t_{2}-t\right)}{1+\delta t_{2}}\right] } \\
& \times \frac{b\left(t-t_{1}\right)}{\delta}+\frac{c\left(t-t_{1}\right)}{\delta^{2}}\left[1+\frac{3 \delta\left(t_{1}+t_{2}\right)}{2}\right], \quad t_{1} \leq t \leq t_{1}+t_{2} .
\end{aligned}
$$

The maximum positive inventory is:

$$
\begin{aligned}
I M & =Q_{0} \\
& =\left(a t_{1}+\frac{b t_{1}^{2}}{2}+\frac{c t_{1}^{3}}{3}+\frac{a \alpha t_{1}^{\beta+1}}{\beta+1}+\frac{b \alpha t_{1}^{\beta+2}}{\beta+2}+\frac{c \alpha t_{1}^{\beta+3}}{\beta+3}+2 a \alpha t_{1}^{\beta+1}+b \alpha t_{1}^{\beta+2}+\frac{2 c \alpha t_{1}^{\beta+3}}{3}\right) .
\end{aligned}
$$

The maximum backordered units are:

$$
\begin{aligned}
I B= & -I_{2}\left(t_{1}+t_{2}\right)=-\left[\left\{\frac{a}{\delta}+\frac{b\left[1+\delta\left(t_{1}+t_{2}\right)\right]}{\delta^{2}}+\frac{c\left[1+\delta\left(t_{1}+t_{2}\right)\right]^{2}}{\delta^{3}}\right\} \times \log \left[\frac{1}{1+\delta t_{2}}\right]\right. \\
& \left.+\frac{b t_{2}}{\delta}+\frac{3 c t_{2}^{2}}{2 \delta}+\frac{2 c t_{1} t_{2}}{\delta}+\frac{c t_{2}}{\delta^{2}}\right] .
\end{aligned}
$$

Hence, the order size during $\left[0, t_{1}+t_{2}\right]$ is:

$$
\begin{aligned}
Q= & I M+I B \\
= & {\left[a t_{1}+\frac{b t_{1}^{2}}{2}+\frac{c t_{1}^{3}}{3}+\frac{a \alpha t_{1}^{\beta+1}}{\beta+1}+\frac{b \alpha t_{1}^{\beta+2}}{\beta+2}+\frac{c \alpha t_{1}^{\beta+3}}{\beta+3}+2 a \alpha t_{1}^{\beta+1}+b \alpha t_{1}^{\beta+2}+\frac{2 c \alpha t_{1}^{\beta+3}}{3}\right] } \\
& -\left[\left\{\frac{a}{\delta}+\frac{b\left[1+\delta\left(t_{1}+t_{2}\right)\right]}{\delta^{2}}+\frac{c\left[1+\delta\left(t_{1}+t_{2}\right)\right]^{2}}{\delta^{3}}\right] \times \log \left[\frac{1}{1+\delta t_{2}}\right]\right. \\
& \left.+\frac{b t_{2}}{\delta}+\frac{3 c t_{2}^{2}}{2 \delta}+\frac{2 c t_{1} t_{2}}{\delta}+\frac{c t_{2}}{\delta^{2}}\right] .
\end{aligned}
$$

The Holding cost during the time interval $\left[0, t_{1}\right]$ is:

$$
\begin{aligned}
& H C=\int_{0}^{t_{1}} h(t) Q_{1}(t) d t=\int_{0}^{t_{1}}(h+\gamma t) Q_{1}(t) d t \\
& \Rightarrow H C=\int_{0}^{t_{1}}(h+\gamma t) e^{-\alpha t^{\beta}} \int_{t}^{t_{1}}\left(a+b u+c u^{2}\right) e^{-\alpha u^{\beta}} d u d t .
\end{aligned}
$$


Since, $\alpha<<1$ so taking the first two terms from the series expansion of the exponential function and then integrating we get:

$$
\begin{aligned}
H C= & a h t_{1}^{2}+\frac{b h t_{1}^{3}}{2}+\frac{c h t_{1}^{4}}{3}+\frac{a h \alpha t_{1}^{\beta+2}}{\beta+1}+\frac{b h \alpha t_{1}^{\beta+3}}{\beta+2} \\
& +\frac{c h \alpha t_{1}^{\beta+4}}{\beta+3}+\frac{\gamma a t_{1}^{3}}{2}+\frac{\gamma b t_{1}^{4}}{2}+\frac{\gamma c t_{1}^{5}}{3}+\frac{\gamma a \alpha t_{1}^{\beta+3}}{\beta+1} \\
& +\frac{\gamma b \alpha t_{1}^{\beta+4}}{\beta+2}+\frac{\gamma c \alpha t_{1}^{\beta+5}}{\beta+3}-\frac{h a \alpha t_{1}^{\beta+2}}{\beta+1}-\frac{h b \alpha t_{1}^{\beta+3}}{2} \\
& -\frac{h c \alpha t_{1}^{\beta+4}}{3}-\frac{\gamma a \alpha t_{1}^{\beta+3}}{\beta+2}-\frac{\gamma b \alpha t_{1}^{\beta+4}}{2(\beta+2)}-\frac{\gamma c \alpha t_{1}^{\beta+5}}{3(\beta+2)} .
\end{aligned}
$$

The total shortage cost during interval $\left[t_{1}, t_{1}+t_{2}\right]$ is:

$$
\begin{aligned}
S C= & c_{2} \int_{t_{1}}^{t_{1}+t_{2}}-Q_{2}(t) d t \\
\Rightarrow S C= & c_{2}\left[\left\{\frac{a}{\delta}+\frac{b\left[1+\delta\left(t_{1}+t_{2}\right)\right]}{\delta^{2}}+\frac{c\left[1+\delta\left(t_{1}+t_{2}\right)\right]^{2}}{\delta^{3}}\right\} \times\left\{t_{2}+\frac{1}{\delta} \log \left[\frac{1}{1+\delta t_{2}}\right]\right\}\right. \\
& \left.-\frac{t_{2}^{2}}{6 \delta^{2}}\left[3 b \delta+2 c \delta\left(3 t_{1}+2 t_{2}\right)+c\right]\right] .
\end{aligned}
$$

Due to stock out during $\left(t_{1}, t_{1}+t_{2}\right)$, shortage is accumulated, but not all customers are willing to wait for the next lot size to arrive. Hence, this results in some loss of sale which accounts to loss in profit.

Lost sale cost is calculated as follows:

$$
\begin{aligned}
L S C= & s \int_{t_{1}}^{T}\left[1-\frac{1}{1+\delta\left(t_{1}+t_{2}-t\right)}\right]\left(a+b t+c t^{2}\right) d t \\
= & s\left[\delta t_{2}+b\left(t_{1} t_{2}+\frac{t_{2}^{2}}{2}+\frac{t_{2}}{\delta}\right)+c\left(t_{1}^{2} t_{2}+t_{1} t_{2}^{2}+\frac{t_{2}^{3}}{2}+\frac{2 t_{1} t_{2}}{\delta}+\frac{t_{2}}{\delta^{2}}\right)\right. \\
& +\left\{\frac{a}{\delta}+\frac{b\left[1+\delta\left(t_{1}+t_{2}\right)\right]}{\delta^{2}}+\frac{c\left[1+\delta\left(t_{1}+t_{2}\right)\right]^{2}}{\delta^{3}}\right\} \times\left\{\frac{1}{\delta} \log \left[\frac{1}{1+\delta t_{2}}\right]\right\} .
\end{aligned}
$$

Purchase cost is as follows:

$$
\begin{aligned}
P C= & c_{1} Q=c_{1}\left[a t_{1}+\frac{b t_{1}^{2}}{2}+\frac{c t_{1}^{3}}{3}+\frac{a \alpha t_{1}^{\beta+1}}{\beta+1}+\frac{b \alpha t_{1}^{\beta+2}}{\beta+2}+\frac{c \alpha t_{1}^{\beta+3}}{\beta+3}\right. \\
& \left.+2 a \alpha t_{1}^{\beta+1}+b \alpha t_{1}^{\beta+2}+\frac{2 c \alpha t_{1}^{\beta+3}}{3}\right] \\
& -c_{1}\left[\left\{\frac{a}{\delta}+\frac{b\left[1+\delta\left(t_{1}+t_{2}\right)\right]}{\delta^{2}}+\frac{c\left[1+\delta\left(t_{1}+t_{2}\right)\right]^{2}}{\delta^{3}}\right\} \times \log \left[\frac{1}{1+\delta t_{2}}\right]\right. \\
& \left.+\frac{b t_{2}}{\delta}+\frac{3 c t_{2}^{2}}{2 \delta}+\frac{2 c t_{1} t_{2}}{\delta}+\frac{c t_{2}}{\delta^{2}}\right] .
\end{aligned}
$$


Total average cost is as follows:

$$
\begin{aligned}
& T A C=\frac{1}{T}[O C+H C+S C+L S C+P C] \\
& =\frac{1}{t_{1}+t_{2}}\left[A+\left[a h t_{1}^{2}+\frac{b h t_{1}^{3}}{2}+\frac{c h t_{1}^{4}}{3}+\frac{a h \alpha t_{1}^{\beta+2}}{\beta+1}+\frac{b h \alpha t_{1}^{\beta+3}}{\beta+2}\right.\right. \\
& +\frac{c h \alpha t_{1}^{\beta+4}}{\beta+3}+\frac{\gamma a t_{1}^{3}}{2}+\frac{\gamma b t_{1}^{4}}{2}+\frac{\gamma c t_{1}^{5}}{3} \\
& +\frac{\gamma a \alpha t_{1}^{\beta+3}}{\beta+1}+\frac{\gamma b \alpha t_{1}^{\beta+4}}{\beta+2}+\frac{\gamma c \alpha t_{1}^{\beta+5}}{\beta+3}-\frac{h a \alpha t_{1}^{\beta+2}}{\beta+1}-\frac{h b \alpha t_{1}^{\beta+3}}{2} \\
& -\frac{h c \alpha t_{1}^{\beta+4}}{3}-\frac{\gamma a \alpha t_{1}^{\beta+3}}{\beta+2}-\frac{\gamma b \alpha t_{1}^{\beta+4}}{2(\beta+2)} \\
& \left.-\frac{\gamma c \alpha t_{1}^{\beta+5}}{3(\beta+2)}\right]+c_{2}\left[\left\{\frac{a}{\delta}+\frac{b\left[1+\delta\left(t_{1}+t_{2}\right)\right]}{\delta^{2}}+\frac{c\left[1+\delta\left(t_{1}+t_{2}\right)\right]^{2}}{\delta^{3}}\right\}\right. \\
& \times\left\{t_{2}+\frac{1}{\delta} \log \left[\frac{1}{1+\delta t_{2}}\right]\right\} \\
& \left.-\frac{t_{2}^{2}}{6 \delta^{2}}\left[3 b \delta+2 c \delta\left(3 t_{1}+2 t_{2}\right)+c\right]\right]+s\left[\delta t_{2}+b\left(t_{1} t_{2}+\frac{t_{2}^{2}}{2}+\frac{t_{2}}{\delta}\right)\right. \\
& +c\left(t_{1}^{2} t_{2}+t_{1} t_{2}^{2}+\frac{t_{2}^{3}}{2}+\frac{2 t_{1} t_{2}}{\delta}+\frac{t_{2}}{\delta^{2}}\right) \\
& \left.+\left\{\frac{a}{\delta}+\frac{b\left[1+\delta\left(t_{1}+t_{2}\right)\right]}{\delta^{2}}+\frac{c\left[1+\delta\left(t_{1}+t_{2}\right)\right]^{2}}{\delta^{3}}\right\} \times\left\{\frac{1}{\delta} \log \left[\frac{1}{1+\delta t_{2}}\right]\right\}\right] \\
& +c_{1}\left[a t_{1}+\frac{b t_{1}^{2}}{2}+\frac{c t_{1}^{3}}{3}+\frac{a \alpha t_{1}^{\beta+1}}{\beta+1}+\frac{b \alpha t_{1}^{\beta+2}}{\beta+2}\right. \\
& \left.+\frac{c \alpha t_{1}^{\beta+3}}{\beta+3}+2 a \alpha t_{1}^{\beta+1}+b \alpha t_{1}^{\beta+2}+\frac{2 c \alpha t_{1}^{\beta+3}}{3}\right] \\
& -c_{1}\left[\left\{\frac{a}{\delta}+\frac{b\left[1+\delta\left(t_{1}+t_{2}\right)\right]}{\delta^{2}}+\frac{c\left[1+\delta\left(t_{1}+t_{2}\right)\right]^{2}}{\delta^{3}}\right\}\right. \\
& \left.\times \log \left[\frac{1}{1+\delta t_{2}}\right]+\frac{b t_{2}}{\delta}+\frac{3 c t_{2}^{2}}{2 \delta}+\frac{2 c t_{1} t_{2}}{\delta}+\frac{c t_{2}}{\delta^{2}}\right] .
\end{aligned}
$$

To minimize the total cost TAC per unit time, the optimal value of $t_{1}$ and $t_{2}$ can be obtained by solving the following equations:

$$
\begin{aligned}
& \frac{\partial(T A C)}{\partial t_{1}}=0 \\
& \Rightarrow-\frac{1}{\left(t_{1}+t_{2}\right)^{2}}\left[A+\left[a h t_{1}^{2}+\frac{b h t_{1}^{3}}{2}+\frac{a \gamma t_{1}^{3}}{2}+\frac{c h t_{1}^{4}}{3}\right.\right. \\
& \quad+\frac{b \gamma t_{1}^{4}}{2}+\frac{c \gamma t_{1}^{5}}{3}-\frac{b h \alpha t_{1}^{\beta+3}}{2}+\frac{b h \alpha t_{1}^{\beta+3}}{\beta+2}+\frac{a \alpha \gamma t_{1}^{\beta+3}}{\beta+1}
\end{aligned}
$$




$$
\begin{aligned}
& \left.-\frac{a \alpha \gamma t_{1}^{\beta+3}}{\beta+2} \frac{c h \alpha t_{1}^{\beta+4}}{3}+\frac{c h \alpha t_{1}^{\beta+4}}{\beta+3}+\frac{b \alpha \gamma t_{1}^{\beta+4}}{2(\beta+2)}-\frac{c \alpha \gamma t_{1}^{\beta+5}}{3(\beta+2)}+\frac{d \alpha \gamma t_{1}^{\beta+5}}{\beta+3}\right] \\
& +\frac{1}{t_{1}+t_{2}}\left[2 a h t_{1}+\frac{3 b h t_{1}^{2}}{2}+\frac{3 a \gamma t_{1}^{2}}{2}\right. \\
& +\frac{4 c h t_{1}^{3}}{3}+2 b \gamma t_{1}^{3}+\frac{5 c \gamma t_{1}^{4}}{3}-\frac{b h \alpha(\beta+3) t_{1}^{\beta+2}}{2}+\frac{b h \alpha(\beta+3) t_{1}^{\beta+2}}{\beta+2} \\
& +\frac{a \alpha(\beta+3) \gamma t_{1}^{\beta+2}}{\beta+1}-\frac{a \alpha(\beta+3) \gamma t_{1}^{\beta+2}}{\beta+2}-\frac{\operatorname{ch} \alpha(\beta+4) t_{1}^{\beta+3}}{3} \\
& \left.+\frac{c h \alpha(\beta+4) t_{1}^{\beta+3}}{\beta+3}+\frac{b \alpha(\beta+4) t_{1}^{\beta+3}}{2(\beta+2)}-\frac{c \alpha(\beta+5) \gamma t_{1}^{\beta+4}}{3(\beta+2)}+\frac{c \alpha(\beta+5) \gamma t_{1}^{\beta+4}}{\beta+3}\right] \\
& +\frac{c_{1}}{t_{1}+t_{2}}\left[a+b t_{1}+c t_{1}^{2}+a \alpha t_{1}^{\beta}+2 a \alpha t_{1}^{\beta}+2 a \alpha(\beta+1) t_{1}^{\beta}+b \alpha t_{1}^{\beta+1}+b \alpha(\beta+2) t_{1}^{\beta+1}\right. \\
& \left.+c \alpha t_{1}^{\beta+2}+\frac{2 c \alpha(\beta+3) t_{1}^{\beta+2}}{3}-\frac{2 c t_{2}}{\delta}-\log \left[\frac{1}{1+\delta t_{2}}\right]\left[\frac{b}{\delta}+\frac{2 c\left(1+\delta\left(t_{1}+t_{2}\right)\right.}{\delta^{2}}\right]\right] \\
& +\frac{s}{t_{1}+t_{2}}\left[b t_{2}+c\left(\frac{2 t_{2}}{\delta}+2 t_{1} t_{2}+t_{1}^{2}\right)+\log \left[\frac{1}{1+\delta t_{2}}\right]\left\{\frac{b}{\delta}+\frac{2 c\left[1+\delta\left(t_{1}+t_{2}\right)\right]}{\delta^{2}}\right\}\right] \\
& -\frac{c_{1}}{\left(t_{1}+t_{2}\right)^{2}}\left[a t_{1}+\frac{b t_{1}^{2}}{2}+\frac{c t_{1}^{3}}{3}+2 a \alpha t_{1}^{\beta+1}+\frac{a \alpha t_{1}^{\beta+1}}{\beta+1}+b \alpha t_{1}^{\beta+2}+\frac{b \alpha t_{1}^{\beta+2}}{\beta+2}+\right. \\
& +\frac{2 c \alpha t_{1}^{\beta+3}}{3}+\frac{c \alpha t_{1}^{\beta+3}}{\beta+3}-\frac{c t_{2}}{\delta^{2}}-\frac{b t_{2}}{\delta}-\frac{2 c t_{1} t_{2}}{\delta}-\frac{3 c t_{1}^{2}}{2 \delta} \\
& \left.-\log \left[\frac{1}{1+\delta t_{2}}\right]\left\{\frac{a}{\delta}+\frac{b\left[1+\delta\left(t_{1}+t_{2}\right)\right]}{\delta^{2}}+\frac{c\left[1+\delta\left(t_{1}+t_{2}\right)\right]^{2}}{\delta^{3}}\right\}\right] \\
& -\frac{s}{\left(t_{1}+t_{2}\right)^{2}}\left[\delta t_{2}+b\left(t_{1} t_{2}+\frac{t_{2}^{2}}{2}+\frac{t_{2}}{\delta}\right)+c\left(t_{1}^{2} t_{2}+t_{1} t_{2}^{2}+\frac{t_{2}^{3}}{2}+\frac{2 t_{1} t_{2}}{\delta}+\frac{t_{2}}{\delta^{2}}\right)\right. \\
& \left.+\left\{\frac{a}{\delta}+\frac{b\left[1+\delta\left(t_{1}+t_{2}\right)\right]}{\delta^{2}}+\frac{c\left[1+\delta\left(t_{1}+t_{2}\right)\right]^{2}}{\delta^{3}}\right\} \times\left\{\frac{1}{\delta} \log \left[\frac{1}{1+\delta t_{2}}\right]\right\}\right]-\frac{c_{2}}{\left(t_{1}+t_{2}\right)^{2}} \\
& \times\left[-\frac{t_{2}^{2}\left(c+3 b \delta+2 b \delta\left(3 t_{1}+2 t_{2}\right)\right)}{6 \delta^{2}}+\left[t_{2}+\frac{a}{\delta} \log \left[\frac{1}{1+\delta}\right]\right]\right. \\
& \times\left\{\frac{a}{\delta}+\frac{b\left[1+\delta\left(t_{1}+t_{2}\right)\right]}{\delta^{2}}+\frac{c\left[1+\delta\left(t_{1}+t_{2}\right)\right]^{2}}{\delta^{3}}\right\}=0
\end{aligned}
$$

and

$$
\begin{aligned}
& \frac{\partial(T A C)}{\partial t_{2}}=0 \\
& \Rightarrow-\frac{1}{\left(t_{1}+t_{2}\right)^{2}}\left[A+\left[a h t_{1}^{2}+\frac{b h t_{1}^{3}}{2}+\frac{c h t_{1}^{4}}{3}+\frac{a h \alpha t_{1}^{\beta+2}}{\beta+1}+\frac{b h \alpha t_{1}^{\beta+3}}{\beta+2}\right.\right. \\
& \quad+\frac{c h \alpha t_{1}^{\beta+4}}{\beta+3}+\frac{\gamma a t_{1}^{3}}{2}+\frac{\gamma b t_{1}^{4}}{2}+\frac{\gamma c t_{1}^{5}}{3}
\end{aligned}
$$




$$
\begin{aligned}
& +\frac{\gamma a \alpha t_{1}^{\beta+3}}{\beta+1}+\frac{\gamma b \alpha t_{1}^{\beta+4}}{\beta+2}+\frac{\gamma c \alpha t_{1}^{\beta+5}}{\beta+3}-\frac{h a \alpha t_{1}^{\beta+2}}{\beta+1}-\frac{h b \alpha t_{1}^{\beta+3}}{2} \\
& \left.-\frac{h c \alpha t_{1}^{\beta+4}}{3}-\frac{\gamma a \alpha t_{1}^{\beta+3}}{\beta+2}-\frac{\gamma b \alpha t_{1}^{\beta+4}}{2(\beta+2)}-\frac{\gamma c \alpha t_{1}^{\beta+5}}{3(\beta+2)}\right] \\
& -\frac{c_{1}}{\left(t_{1}+t_{2}\right)^{2}}\left[a t_{1}+\frac{b t_{1}^{2}}{2}+\frac{c t_{1}^{3}}{3}+2 a \alpha t_{1}^{\beta+1}+\frac{a \alpha t_{1}^{\beta+1}}{\beta+1}\right. \\
& +b \alpha t_{1}^{\beta+2}+\frac{b \alpha t_{1}^{\beta+2}}{\beta+2}+\frac{c \alpha t_{1}^{\beta+3}}{\beta+3}+\frac{2 c \alpha t_{1}^{\beta+3}}{3}-\frac{c t_{2}}{\delta^{2}}-\frac{b t_{2}}{\delta}-\frac{2 c t_{1} t_{2}}{\delta} \\
& \left.-\frac{2 c t_{2}^{2}}{\delta}-\frac{3 c t_{2}^{2}}{2 \delta}-\left\{\frac{a}{\delta}+\frac{b\left[1+\delta\left(t_{1}+t_{2}\right)\right]}{\delta^{2}}+\frac{c\left[1+\delta\left(t_{1}+t_{2}\right)\right]^{2}}{\delta^{3}}\right\} \times \log \left[\frac{1}{1+\delta t_{2}}\right]\right] \\
& -\frac{s}{\left(t_{1}+t_{2}\right)^{2}}\left[\delta t_{2}+b\left(t_{1} t_{2}+\frac{t_{2}^{2}}{2}+\frac{t_{2}}{\delta}\right)+c\left(t_{1}^{2} t_{2}+t_{1} t_{2}^{2}+\frac{t_{2}^{3}}{2}+\frac{2 t_{1} t_{2}}{\delta}+\frac{t_{2}}{\delta^{2}}\right)\right. \\
& \left.+\left\{\frac{a}{\delta}+\frac{b\left[1+\delta\left(t_{1}+t_{2}\right)\right]}{\delta^{2}}+\frac{c\left[1+\delta\left(t_{1}+t_{2}\right)\right]^{2}}{\delta^{3}}\right\} \times\left\{\frac{1}{\delta} \log \left[\frac{1}{1+\delta t_{2}}\right]\right\}\right]-\frac{c_{2}}{\left(t_{1}+t_{2}\right)^{2}} \\
& \times\left[\left\{\frac{a}{\delta}+\frac{b\left[1+\delta\left(t_{1}+t_{2}\right)\right]}{\delta^{2}}+\frac{c\left[1+\delta\left(t_{1}+t_{2}\right)\right]^{2}}{\delta^{3}}\right\}\left\{t_{2}+\frac{a}{\delta} \log \left[\frac{1}{1+\delta t_{2}}\right]\right\}\right. \\
& \left.-\frac{t_{2}^{2}\left(c+3 b \delta+2 c \delta\left(3 t_{1}+2 t_{2}\right)\right)}{6 \delta^{2}}\right] \\
& \times \frac{s}{t_{1}+t_{2}}\left[\delta+b\left(\frac{1}{\delta}+t_{1}+t_{2}\right)+c\left(\frac{1}{\delta^{2}}+\frac{2 t_{1}}{\delta}+t_{1}^{2}+2 t_{1} t_{2}+t_{2}^{2}\right)\right. \\
& +\log \left[\frac{1}{1+\delta t_{2}}\right]+\frac{b}{\delta}+\frac{1}{\delta^{2}}\left(2 c\left(1+\delta\left(t_{1}+t_{2}\right)\right)\right] \\
& \left.-\frac{\delta}{1+\delta t_{2}}\left\{\frac{a}{\delta}+\frac{b\left[1+\delta\left(t_{1}+t_{2}\right)\right]}{\delta^{2}}+\frac{c\left[1+\delta\left(t_{1}+t_{2}\right)\right]^{2}}{\delta^{3}}\right\}\right] \\
& +\frac{c_{1}}{t_{1}+t_{2}}\left[-\frac{c}{\delta^{2}}-\frac{b}{\delta}-\frac{2 c t_{1}}{\delta}-\frac{3 c t_{2}}{\delta}\right. \\
& -\log \left[\frac{1}{1+\delta t_{2}}\right]+\frac{b}{\delta}+\frac{1}{\delta^{2}}\left(2 c\left(1+\delta\left(t_{1}+t_{2}\right)\right)\right] \\
& \left.+\frac{\delta}{1+\delta t_{2}}\left\{\frac{a}{\delta}+\frac{b\left[1+\delta\left(t_{1}+t_{2}\right)\right]}{\delta^{2}}+\frac{c\left[1+\delta\left(t_{1}+t_{2}\right)\right]^{2}}{\delta^{3}}\right\}\right] \\
& +\frac{c_{2}}{t_{1}+t_{2}}\left[-\frac{2 c t_{2}^{2}}{3 \delta}-\frac{t_{2}\left(c+3 b \delta+2 c \delta\left(3 t_{1}+2 t_{2}\right)\right)}{3 \delta^{2}}+\left[\frac{a}{\delta} \log \left[\frac{1}{1+\delta t_{2}}\right]+t_{2}\right]\right. \\
& \times\left[\frac{b}{\delta}+\frac{2 c\left[1+\delta\left(t_{1}+t_{2}\right)\right]}{\delta^{2}}\right]+\left(1-\frac{a}{1+\delta t_{2}}\right) \\
& \left.\times\left\{\frac{a}{\delta}+\frac{b\left[1+\delta\left(t_{1}+t_{2}\right)\right]}{\delta^{2}}+\frac{c\left[1+\delta\left(t_{1}+t_{2}\right)\right]^{2}}{\delta^{3}}\right\}\right]=0
\end{aligned}
$$


Providing that Eqs. (14) and (15) satisfies the following conditions:

$$
\left(\frac{\partial^{2}(T A C)}{\partial t_{1}^{2}}\right)\left(\frac{\partial^{2}(T A C)}{\partial T^{2}}\right)-\left(\frac{\partial^{2}(T A C)}{\partial t_{1} \partial T}\right)^{2}>0, \quad\left(\frac{\partial^{2}(T A C)}{\partial t_{1}^{2}}\right)>0 \text { and } \frac{\partial^{2}(T A C)}{\partial T^{2}}>0 .
$$

The Eqs. (14) and (15) are highly nonlinear. They can be solved by Mathematica-9.0 software for a given set of known parameters. The obtained values of $t_{1}$ and $t_{2}$ must satisfy the Eq. (13) to minimize the total average cost per time unit of the inventory system. The total average cost function is highly nonlinear, thus the convexity of the function is shown graphically in "Graphical Proof for the Convexity of the Total Cost Function" section. To illustrate this we have given a numerical example and a sensitivity analysis in the following sections.

\section{Numerical Example}

The proposed models are illustrated below by considering the following examples.

Example 1 The following numerical values of the parameter in proper unit were considered as input for numerical and graphical analysis of the model, $A=80, a=0.8, b=0.6, c=$ $0.4, c_{1}=0.2, c_{2}=0.4, \alpha=0.4, \beta=4, \gamma=0.4, \delta=0.3, s=0.3$ and $h=0.6$. The values of different parameters considered here are realistic, though these are not taken from any case study. Then the optimal solution is $t_{1}^{*}=1.16952, t_{2}^{*}=4.72713$. For these values of $t_{1}$ and $t_{2}$, the second order derivative found to be $\frac{\partial^{2}(T A C)}{\partial t_{1}^{2}}=3.02049>0$ and $\frac{\partial^{2}(T A C)}{\partial t_{2}^{2}}=$ $1.08052>0, \frac{\partial^{2}(T A C)}{\partial t_{1} \partial t_{2}}=1.00194$ and $\frac{\partial^{2}(T A C)}{\partial t_{1}^{2}} \times \frac{\partial^{2}(T A C)}{\partial t_{2}^{2}}-\left(\frac{\partial^{2}(T A C)}{\partial t_{1} \partial T}\right)^{2}=2.02246>0$. So the values of $t_{1}^{*}$ and $t_{2}^{*}$ are minimize the total average cost. Based on these input data, the computed outputs $Q^{*}=1.12811$ and $T A C^{*}=23.4766$.

\section{Graphical Proof for the Convexity of the Total Cost Function}

If we plot the total cost function (13) with some values of $t_{1}$ and $t_{2}$ such that $t_{1}$ is $0.6-1.7$ and $t_{2}$ is $2-9$ then we get strictly convex graph of total cost function TAC given by the Fig. 5 . From Fig. 5, we observe that the optimal replenishment schedule uniquely exists and the total average cost to the inventory system is a convex function. The convexity of the total cost function TAC is shown in Fig. 3 with respect to $t_{1}$ fixed at $t_{2}=4.72713$. The convexity of the total cost function TAC is shown in Fig. 4 with respect to $t_{2}$ fixed at $t_{1}=1.16952$. The observation from Figs. 3 and 4, the total average cost function is a strictly convex function. Thus, the optimum value of $t_{1}$ and $t_{2}$ can be obtained with the help of the total average cost function of the model provided that the total inventory cost per unit time of the inventory system is minimized (Fig. 5).

\section{Case Study}

From Example 1, When $\alpha=0$, i.e. the deterioration of the item is not considered and the values of all other parameters remain the same, $t_{1}^{*}=1.137932, t_{2}^{*}=4.51788$ and the minimum total cost $T A C^{*}=23.38$ is $0.4115 \%$ less than that value when $\alpha=0.4$. If $\beta=1$, 


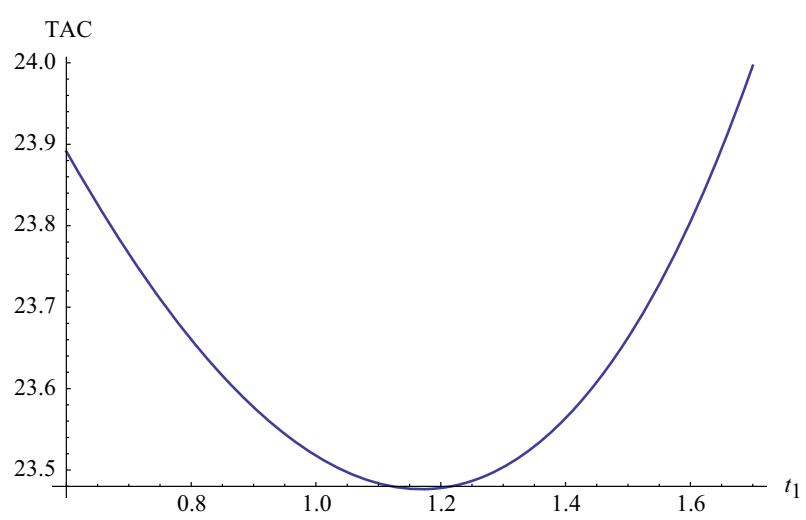

Fig. $3 T A C$ vs. $t_{1}$ at $t_{2}=4.72713$

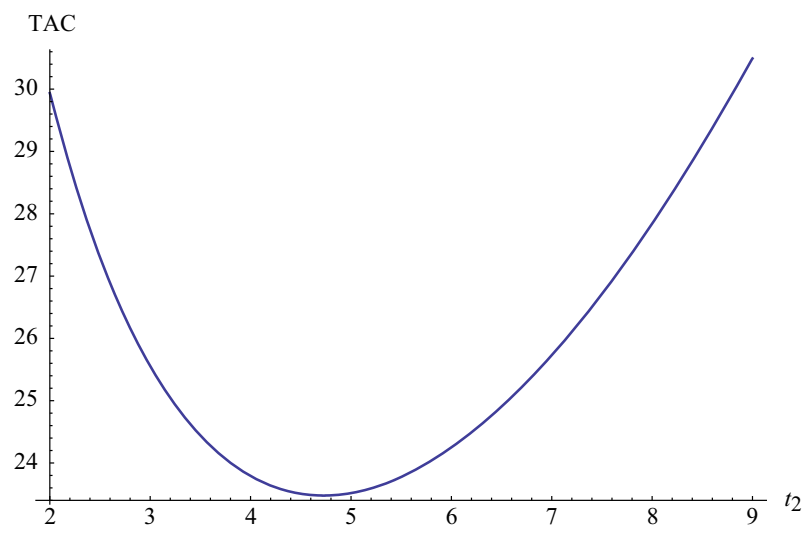

Fig. $4 T A C$ vs. $t_{2}$ at $t_{1}=1.16952$

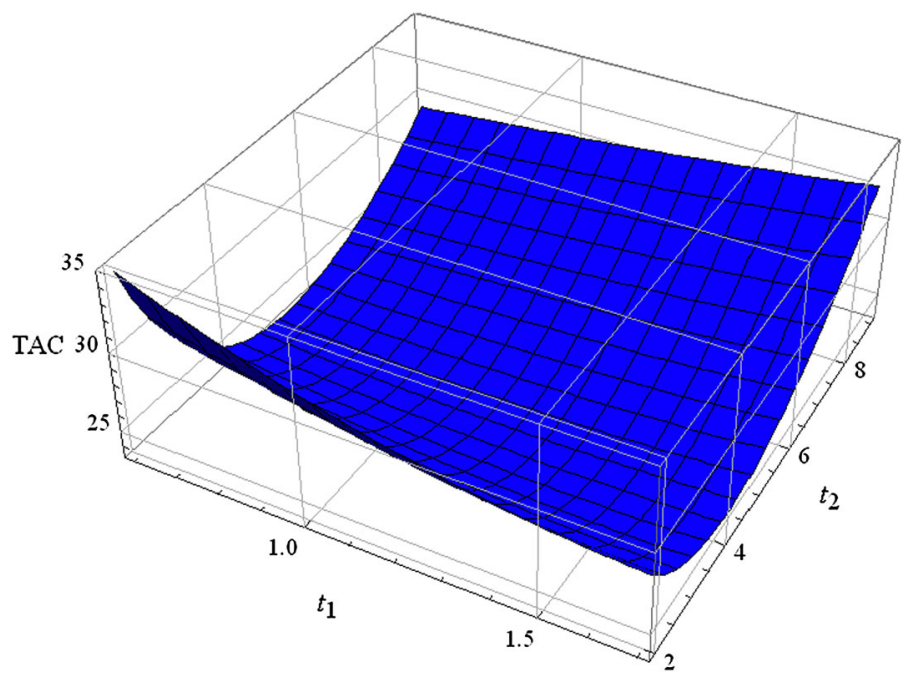

Fig. $5 T A C$ vs. $t_{1}$ and $t_{2}$ 
Table 2 Sensitivity analysis based on changes of parameters

\begin{tabular}{|c|c|c|c|c|c|}
\hline Parameter & $\%$ Change & $t_{1}^{*}$ & $t_{2}^{*}$ & $T A C^{*}$ & (\%) Change in $T A C^{*}$ \\
\hline \multirow[t]{4}{*}{$A$} & +50 & 1.20223 & 5.61633 & 29.758 & 26.756 \\
\hline & +25 & 1.18745 & 5.20233 & 26.7306 & 13.8606 \\
\hline & -25 & 1.14662 & 4.16204 & 19.9098 & -15.193 \\
\hline & -50 & 1.11466 & 3.4482 & 15.8644 & -32.4246 \\
\hline \multirow[t]{4}{*}{$a$} & +50 & 1.73806 & 2.87735 & 31.9081 & 35.9145 \\
\hline & +25 & 0.48494 & 3.69917 & 30.2394 & 28.8066 \\
\hline & -25 & 0.479443 & 6.35384 & 17.6235 & -24.9316 \\
\hline & -50 & 0.443298 & 8.70754 & 10.8644 & -54.4606 \\
\hline \multirow[t]{4}{*}{$b$} & +50 & 1.15017 & 4.59613 & 24.4408 & 4.1071 \\
\hline & +25 & 1.15947 & 4.66079 & 23.9619 & 2.0659 \\
\hline & -25 & 1.18039 & 4.79515 & 22.9846 & -2.0957 \\
\hline & -50 & 1.19217 & 4.8649 & 22.4859 & -4.2199 \\
\hline \multirow[t]{4}{*}{$c$} & +50 & 1.2364 & 3.95645 & 26.7354 & 13.8811 \\
\hline & +25 & 1.20697 & 4.29596 & 25.1888 & 7.2932 \\
\hline & -25 & 1.12053 & 5.30585 & 21.5394 & -8.2516 \\
\hline & -50 & 1.05385 & 6.15705 & 19.2445 & -18.0271 \\
\hline \multirow[t]{4}{*}{$c_{1}$} & +50 & 1.07844 & 4.71734 & 24.0251 & 2.3364 \\
\hline & +25 & 1.11887 & 4.72656 & 23.7544 & 1.1833 \\
\hline & -25 & 1.23782 & 4.71189 & 23.1891 & -1.2246 \\
\hline & -50 & 1.3451 & 4.66044 & 22.8858 & -2.5165 \\
\hline \multirow[t]{4}{*}{$c_{2}$} & +50 & 1.45187 & 3.58315 & 27.9021 & 18.8507 \\
\hline & +25 & 1.33784 & 4.07269 & 25.8522 & 10.119 \\
\hline & -25 & 0.837062 & 5.7293 & 20.6314 & -12.1193 \\
\hline & -50 & 0.0863749 & 7.48302 & 16.9027 & -28.0019 \\
\hline \multirow[t]{4}{*}{$\alpha$} & +50 & 1.11131 & 4.78563 & 23.5052 & 0.1218 \\
\hline & +25 & 1.13836 & 4.75842 & 23.4918 & 0.0647 \\
\hline & -25 & 1.20612 & 4.69043 & 23.4589 & -0.0754 \\
\hline & -50 & 1.25029 & 4.64626 & 23.4381 & -0.164 \\
\hline \multirow[t]{4}{*}{$\beta$} & +50 & 1.11981 & 4.77419 & 23.4826 & 0.0256 \\
\hline & +25 & 1.14328 & 4.75196 & 23.4797 & 0.0132 \\
\hline & -25 & 1.19688 & 4.70133 & 23.4738 & -0.0119 \\
\hline & -50 & 1.22254 & 4.6774 & 23.4728 & -0.0162 \\
\hline \multirow[t]{4}{*}{$\gamma$} & +50 & 1.08896 & 4.80946 & 23.5248 & 0.2053 \\
\hline & +25 & 1.12593 & 4.77163 & 23.5024 & 0.1099 \\
\hline & -25 & 1.22308 & 4.67267 & 23.4458 & -0.1312 \\
\hline & -50 & 1.29394 & 4.6011 & 23.4076 & -0.2939 \\
\hline \multirow[t]{4}{*}{$\delta$} & +50 & 0.473094 & 6.26416 & 19.2566 & -17.9753 \\
\hline & +25 & 0.863775 & 5.2178 & 20.8957 & -10.9858 \\
\hline & -25 & 1.49917 & 3.64649 & 28.3627 & 20.8126 \\
\hline & -50 & 1.90379 & 1.91037 & 38.9295 & 65.8226 \\
\hline \multirow[t]{2}{*}{$s$} & +50 & 0.811419 & 5.24758 & 22.4984 & -4.1667 \\
\hline & +25 & 1.03932 & 4.93388 & 23.0115 & -1.9811 \\
\hline
\end{tabular}


Table 2 continued

\begin{tabular}{lllllc}
\hline Parameter & $\%$ Change & $t_{1}^{*}$ & $t_{2}^{*}$ & $T A C^{*}$ & $(\%)$ Change in $T A C^{*}$ \\
\hline & -25 & 1.26297 & 4.5619 & 23.9097 & 1.8448 \\
& -50 & 1.33615 & 4.42032 & 24.3181 & 3.5844 \\
& +50 & 1.43374 & 4.75677 & 23.6752 & 0.8459 \\
& +25 & 1.14582 & 4.75492 & 23.5136 & 0.1576 \\
& -25 & 1.18917 & 4.70293 & 23.4385 & -0.1623 \\
& -50 & 1.20539 & 4.6818 & 23.3997 & -0.3276 \\
\hline
\end{tabular}

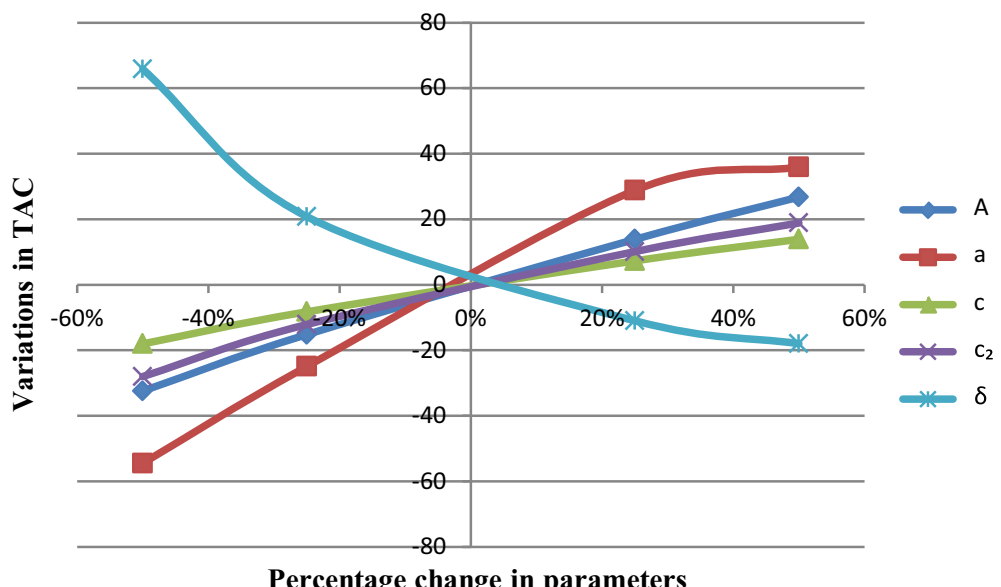

Fig. 6 Behaviour of optimal average total cost

i.e. the deterioration of the item is constant and the values of all other parameters remain the same, $t_{1} *=1.24232, t_{2}^{*}=4.65972$ and the minimum total cost $T A C^{*}=23.4769$ is $0.0013 \%$ higher than that value when $\beta=4$. If $\beta=0.4$, i.e. the deterioration of the item is decreasing and the values of all other parameters remain the same, $t_{1}^{*}=1.24784$, $t_{2}^{*}=4.65602$ and the minimum total cost $T A C^{*}=23.4859$ is $0.396 \%$ higher than that value when $\beta=4$. It is noted that when $\alpha=0$ (i.e. deterioration of the item is not considered at all), the total average cost of the system is less than that under a two parameter Weibull distribution deterioration. But when $\beta=1$ (i.e. deterioration rate of the item is constant), the total average cost of the system is greater than that under a two parameter Weibull distribution deterioration. Therefore, Weibull distribution deterioration is more applicable than constant deterioration.

\section{Sensitivity Analysis}

Based on above Example 1, we have performed sensitivity analysis by changing one parameter at a time by \pm 25 and \pm 50 , and keeping the remaining parameters at their original values. Table 2 summarize the results.

Based on the results of Table 2, the following observations can be made. 


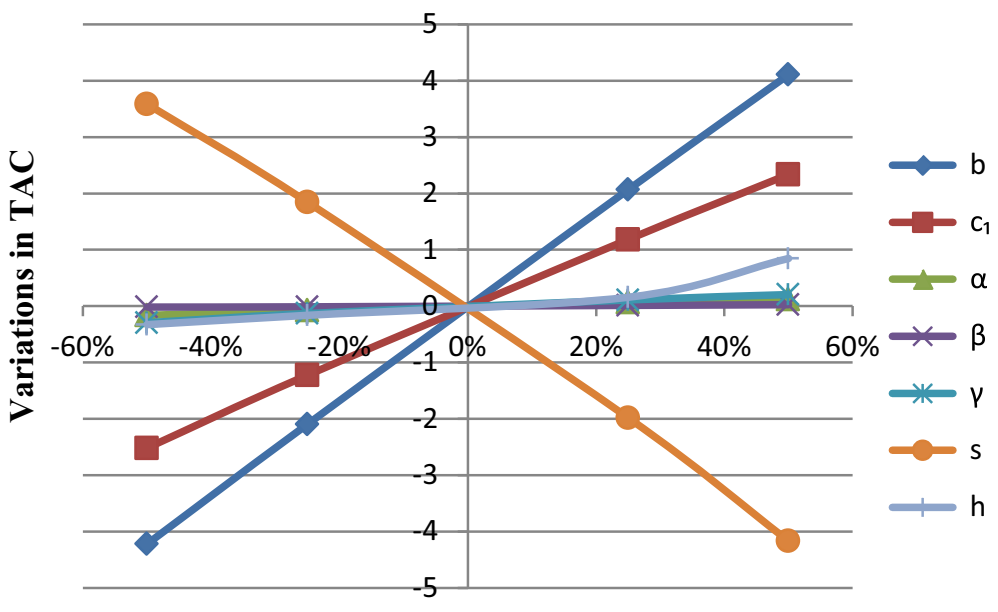

Percentage change in parameters

Fig. 7 Behaviour of optimal average total cost

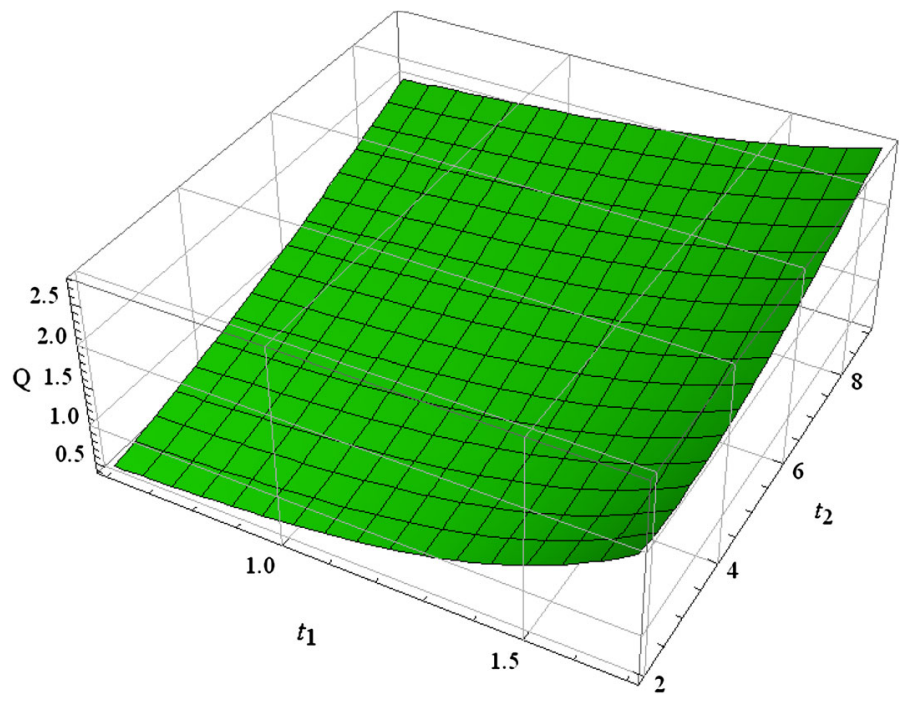

Fig. $8 Q$ vs. $t_{1}$ and $t_{2}$

(i) An increase on the values of the parameters $A, a, b, c, c_{1}$ and $c_{2}$ will result to an increase on $T A C^{*}$ (Fig. 6).

(ii) An increase in the values of the parameter $\delta$ and $s$ will result to an decrease on $T A C^{*}$ (Fig. 7).

(iii) An increase in the values of the parameters $\alpha, \beta, \gamma s$ and $h$ will result in slightly increase in $T A C^{*}$.

The graphical representation of $Q$ vs. $t_{1}$ and $t_{2}$ of the Example 1 is shown in Fig. 8. From Fig. 8, we can observe, when $t_{1}$ and $t_{2}$ increases then $Q$ increase. 


\section{Conclusion}

This paper presents an inventory model of direct application to the business enterprises that consider the fact that the storage item is deteriorated during storage periods and in which the demand, deterioration, and holding cost depend upon the time. In the present paper an economic ordered quantity model has been developed for an item with two parameter Weibull deterioration where shortages are allowed and the demand are partially backlogged. The optimal cycle time and total optimal average cost have been derived from the model. It can be concluded that to minimize the total average cost, it is required to minimize the ordering cost, purchase cost, holding cost, shortage cost, the cost of lost sales per unit, the demand rate, backlogging parameter, scale parameter and shape parameter. From "Case Study" section, it can be observed that Weibull distribution deterioration is more applicable than constant deterioration. From this paper the outcomes show that, (i) Weibull distribution deterioration is more applicable than constant deterioration and makes the scope of its broader application. (ii) When the value of demand factor $a, b$ and $c$ increases and other parameters' values are fixed, it can be observed that the optimal total cost per unit time increase. This implies that when the demand factor $a, b$ and $c$ is increasing, the market demand rate will increase, which in turn make the enterprises increase the order quantity pre replenishment cycle and shorten the replenishment cycle to meet the increasing market need. Moreover the enterprises will make the selling price higher to obtain more profit. (iii) If the backlogging parameter $\delta$ is increased then the total cost will be decreased. That is, in order to minimize the cost, the retailer should increase the backlogging parameter. Sensitivity analysis shows how the different parameters affect the optimal cycle time and total optimal average cost. Finally, the proposed model has been verified by the numerical and graphical analysis. The obtained results indicate the validity and stability of the model. The proposed model can further be enriched by taking more realistic assumptions, such as finite replenishment rate, demand can be considered as a ramp type or trapezoidal type or probabilistic, multi-item inventory models, deterioration can be considered as a three parameter Weibull distribution, etc.

Acknowledgments The author thanks the editor and anonymous reviewers for their valuable and constructive comments, which have led to a significant improvement in the manuscript.

\section{References}

1. Amutha, R.L., Chandrasekaran, E.: An EOQ model for deteriorating items with quadratic demand and time dependent holding cost. Int. J. Emerg. Sci. Eng. 1(5), 5-6 (2013)

2. Cárdenas-Barrón, L.E., Chung, K.J., Treviño-Garza, G.: Celebrating a century of the economic order quantity model in honor of Ford Whitman Harris. Int. J. Prod. Econ. 155, 1-7 (2014)

3. Covert, R.P., Philip, G.C.: An EOQ model for items with Weibull distribution deterioration. AIIE Trans. 5, 323-326 (1973)

4. Deb, M., Chaudhuri, K.S.: An EOQ model for items with finite rate of production and variable rate of deterioration. AIIE Opsearch 23, 175-181 (1986)

5. Geetha, K.V., Udayakumar, R.: Optimal lot sizing policy for non-instantaneous deteriorating items with price and advertisement dependent demand under partial backlogging. Int. J. Appl. Comput. Math, (2015). doi:10.1007/s40819-015-0053-7

6. Ghosh, S.K., Chaudhuri, K.S.: An order level inventory model for a deteriorating item with Weibull deterioration, time-quadratic demand and shortages. Adv. Model. Optim. 6(1), 21-35 (2004)

7. Ghosh, S.K., Khanra, S., Chaudhuri, K.S.: An EOQ model for a deteriorating item with time-varying demand and time-dependent partial backlogging. Int. J. Math. Oper. Res. 3(1), 264-279 (2011) 
8. Giri, B.C., Goswami, A., Chaudhuri, K.S.: An EOQ model for deteriorating items with time varying demand and costs. J. Oper. Res. Soc. 47, 1398-1405 (1996)

9. Kharna, S., Chaudhuri, K.S.: A note on order level inventory model for a deteriorating item with time dependent quadratic demand. Comput. Oper. Res. 30, 901-1916 (2003)

10. Manna, S.K., Chaudhuri, K.S.: An order level inventory model for a deteriorating item with quadratic time-varying demand, shortage and partial backlogging. ARPN J. Eng. Appl. Sci. 9(5), 1-7 (2014)

11. Mishra, U., Tripathy, C.K.: An inventory model for time dependent Weibull deterioration with partial backlogging. Am. J. Oper. Res. 2(2), 11-15 (2012)

12. Mishra, V.K., Singh, L.S.: Deteriorating inventory model with time dependent demand and partial backlogging. Appl. Math. Sci. 4(72), 3611-3619 (2010)

13. Mishra, V.K., Singh, L.S., Kumar, R.: An inventory model for deteriorating items with time dependent demand and time-varying holding cost under partial backlogging. J. Ind. Eng. Int. 9(4), 1-5 (2013)

14. Misra, R.B.: Optimum production lot-size model for a system with deteriorating inventory. Int. J. Prod. Res. 13, 495-505 (1975)

15. Philip, G.C.: A generalized EOQ model for items with Weibull distribution deterioration. AIIE Trans. 6, 159-162 (1974)

16. Pradhan, L.M., Tripathy, C.K.: An EOQ model for three parameter Weibull deteriorating item with partial backlogging. LogForum 9(1), 35-42 (2013)

17. Roy Chowdhury, R., Ghosh, S.K., Chaudhuri, K.S.: An inventory model for deteriorating items with stock and price sensitive demand. Int. J. Appl. Comput. Math. 1, 187-201 (2015). doi:10.1007/s40819-0140011-9

18. Roy, R., Ghosh, S.K., Chaudhuri, K.S.: An order-level inventory model for a deteriorating item with time-quadratic demand and time-dependent partial backlogging with shortages in all cycles. Am. J. Math. Manag. Sci. 33(2), 75-97 (2014)

19. Sana, S.S.: EOQ model of deteriorating and ameliorating items. Math. Comput. Model. 52(1, 2), 284-302 (2010)

20. Sarkar, T., Ghosh, S.K., Chaudhuri, K.S.: An optimal inventory replenishment policy for a deteriorating item with time-quadratic demand and time-dependent partial backlogging with shortages in all cycles. Appl. Math. Comput. 218, 9147-9155 (2012)

21. Shah, N.H., Shukla, K.T.: Deteriorating inventory model for waiting time partial backlogging. Appl. Math. Sci. 3(9), 421-428 (2009)

22. Taleizadeh, A.A., Mohammadi, B., Cárdenas-Barrón, L.E., Samimi, H.: An EOQ model for perishable product with special sale and shortage. Int. J. Prod. Econ. 145(1), 318-338 (2013)

23. Taleizadeh, A.A., Noori-daryan, M., Cárdenas-Barrón, L.E.: Joint optimization of price, replenishment frequency, replenishment cycle and production rate in vendor managed inventory system with deteriorating items. Int. J. Prod. Econ. 159, 285-295 (2015)

24. Tripathy, C.K., Mishra, U.: Ordering policy for Weibull deteriorating items for quadratic demand with permissible delay in payments. Appl. Math. Sci. 4(44), 2181-2191 (2010)

25. Venkateswarlu, R., Mohan, R.: An inventory model with quadratic demand, constant deterioration and salvage value. Res. J. Manag. Sci. 3(1), 18-22 (2013)

26. Widyadana, G.A., Cárdenas-Barrón, L.E., Wee, H.M.: Economic order quantity model for deteriorating items and planned backorder level. Math. Comput. Model. 54(5-6), 1569-1575 (2011) 